\title{
Relationships Between Forced Oscillatory Impedance and 6-minute Walk Distance After Pulmonary Rehabilitation in COPD
}

This article was published in the following Dove Press journal: International Journal of Chronic Obstructive Pulmonary Disease

\author{
Sabine C Zimmermann (D) \\ Cindy Thamrin (D) \\ Andrew SL Chan (iD) ${ }^{2,3}$ \\ Amy Bertolin' \\ David G Chapman (D) 1,4 \\ Gregory G King ${ }^{1-3}$
}

'The Woolcock Emphysema Centre, Woolcock Institute of Medical Research, The University of Sydney, Glebe, NSW 2037, Australia; ${ }^{2}$ The Northern Clinical School, Faculty of Medicine and Health Sciences, The University of Sydney, Camperdown, NSW 2006, Australia; ${ }^{3}$ Department of Respiratory Medicine, Royal North Shore Hospital, St Leonards, NSW 2065, Australia; ${ }^{4}$ School of Life Sciences, Faculty of Science, University of Technology Sydney, Ultimo, NSW 2007, Australia
Rationale: Pulmonary rehabilitation for chronic obstructive pulmonary disease (COPD) reduces dyspnoea and improves exercise capacity and quality of life. The improvement in exercise capacity is variable and unpredictable, however. Respiratory system impedance obtained by forced oscillation technique (FOT) as a measure of ventilatory impairment in COPD may relate to improvement in exercise capacity with pulmonary rehabilitation. We aimed to determine if baseline FOT parameters relate to changes in exercise capacity following pulmonary rehabilitation.

Methods: At the start of rehabilitation, 15 COPD subjects (mean(SD) 75.2(6.1) years, FEV1 z-score $-2.61(0.84)$ ) had measurements by FOT, spirometry, plethysmographic lung volumes and 6-minute walk distance (6MWD). Respiratory system resistance (Rrs) and reactance (Xrs) parameters as the mean over all breaths $\left(\mathrm{R}_{\text {mean }}, \mathrm{X}_{\text {mean }}\right)$, during inspiration only $\left(\mathrm{R}_{\mathrm{insp}}\right.$, $X_{\text {insp }}$ ), and expiratory flow limitation (DeltaXrs $=X_{\text {insp }}-X_{\text {exp }}$ ), were calculated. FOT and $6 \mathrm{MWD}$ measurements were repeated at completion of rehabilitation and 3 months after completion.

Results: At baseline, Xrs measures were unrelated to 6MWD. $\mathrm{X}_{\text {insp }}$ improved significantly with rehabilitation (from mean(SD) $-2.35(1.02)$ to $-2.04(0.85) \mathrm{cmH}_{2}$ O.s.L ${ }^{-1}, \mathrm{p}=0.008$ ), while other FOT parameters did not. No FOT parameters related to the change in $6 \mathrm{MWD}$ at program completion. Baseline $\mathrm{X}_{\text {mean, }}$ DeltaXrs, and FVC z-score correlated with the change in 6MWD between completion and 3 months after completion of rehabilitation $\left(\mathrm{r}_{\mathrm{s}}\right.$ $=0.62, \mathrm{p}=0.03 ; \mathrm{r}_{\mathrm{s}}=-0.65, \mathrm{p}=0.02$; and $\mathrm{r}_{\mathrm{s}}=0.62, \mathrm{p}=0.03$, respectively); with worse ventilatory impairment predicting loss of 6MWD. There were no relationships between Rrs parameters, FEV1 or FEV1/FVC z-scores and changes in 6MWD.

Conclusion: Baseline reactance parameters may be helpful in predicting those patients with COPD at most risk of loss of exercise capacity following completion of pulmonary rehabilitation.

Keywords: COPD, forced oscillation technique, reactance, pulmonary rehabilitation, sixminute walk test, quality of life

\section{Introduction}

Pulmonary rehabilitation for chronic obstructive pulmonary disease (COPD) reduces dyspnoea and improves exercise capacity and quality of life. ${ }^{1,2}$ The improvement in exercise capacity can be variable and patients often struggle to maintain regular exercise after completing the rehabilitation program. Improvements in 6-minute walk distance (6MWD) and health-related quality of
Correspondence: Sabine C Zimmermann The Woolcock Institute of Medical Research, 43I Glebe Point Road, Glebe, NSW 2037, Australia

Tel +6I $291 \mid 40404$

Fax +61291140010

Email sabine.zimmermann@sydney.edu.au 
life tend to be greater when their respective baseline impairments are worse and when spirometry is more impaired. $^{3-5}$ However, there is still a need to find additional predictors of response to pulmonary rehabilitation, which may add mechanistic understanding of the response to rehabilitation and thereby be potentially useful for tailoring programs in COPD patients.

COPD is characterised by airway narrowing and expiratory airway collapse resulting in expiratory flow limitation (EFL), gas trapping and hyperinflation. These functional abnormalities cause exertional dyspnoea, reduced exercise capacity and poor quality of life. ${ }^{6-8}$ Spirometry relates to morbidity and mortality in COPD patients but other functional measurements that reflect the aforementioned mechanical derangements will be clinically relevant, if they relate to exercise capacity and quality of life. In this regard, the forced oscillation technique (FOT) as an effort-independent measurement of respiratory system impedance, may provide clinically useful information in relation to pulmonary rehabilitation for COPD patients because FOT impedance is related to lung hyperinflation ${ }^{9}$ and is sensitive to EFL. ${ }^{10}$

Respiratory system impedance has components of resistance (Rrs) and reactance (Xrs), which reflect airway calibre and elastic properties of the respiratory system under oscillatory conditions, respectively. Both Rrs and Xrs are sensitive to airway narrowing and closure, and also relate to hyperinflation in COPD; with their responses to bronchodilator also being sensitive to decreases in hyperinflation and gas trapping. ${ }^{9}$ Dynamic airway collapse during the expiratory phase of tidal breathing may cause expiratory flow limitation. The expiratory decrease in Xrs (indicating increased oscillatory lung stiffness) is a highly sensitive and specific marker of EFL in $\mathrm{COPD}^{10}$ and is clinically important because it is associated with greater symptoms, worse quality of life, and greater gas trapping and hyperinflation. ${ }^{11}$

In addition to pharmacological treatment, pulmonary rehabilitation also leads to a decrease in resting hyperinflation $^{12}$ and a reduction in breathing rate at a given exercise intensity. ${ }^{12,13}$ Because Xrs parameters correlate with airway calibre, hyperinflation, dynamic airway collapse and EFL (all of which cause ventilatory limitation that impairs exercise capacity), they may also relate to exercise capacity, and to the change in exercise capacity as a result of pulmonary rehabilitation.

We therefore conducted an exploratory pilot study to assess the relationships between baseline FOT indices and
6MWD at baseline and its change following a pulmonary rehabilitation program. We hypothesised that in COPD, the severity of baseline ventilatory impairment measured by FOT parameters relates to $6 \mathrm{MWD}$ and to the change in 6MWD after pulmonary rehabilitation. Our secondary aims were to examine those same relationships with spirometry and hyperinflation.

\section{Methods}

\section{Study Design}

This was an exploratory, prospective observational study of COPD patients attending the pulmonary rehabilitation service at the Royal North Shore Hospital in Sydney, Australia. Participants underwent measurements of FOT, spirometry, lung volumes, quality of life (assessed by St. George's Respiratory Questionnaire, SGRQ) and a six-minute walk test (6MWT) at their initial assessment for the rehabilitation program. FOT, SGRQ and 6MWT were repeated at the conclusion of the program and 3 months following the completion of rehabilitation. Approval was obtained from the Human Research Ethics Committee of the Northern Sydney Local Health District (LNR/16/HAWKE/11). The study was conducted in accordance with the Declaration of Helsinki.

\section{Subjects}

Twenty-one subjects who were about to start pulmonary rehabilitation and had a physician diagnosis of COPD according to Australian guidelines, ${ }^{14}$ were enrolled in this study. However, 15 of those subjects completed the pulmonary rehabilitation program and completed at least 14 of the 16 scheduled sessions; and were included in this analysis. All subjects had at least 10 pack-years history of cigarette smoking and had airflow obstruction on post-bronchodilator spirometry. Exclusion criteria were the presence of any chronic respiratory condition other than COPD, exacerbation within the last 4 weeks, any condition that limited exercise other than COPD such as orthopaedic comorbidity, or any significant cardiac disease such as angina or heart failure. All participants provided written informed consent.

\section{Lung Function Tests}

Spirometry and lung volumes were measured at enrolment in a body plethysmograph (Sensormedics, California) according to ATS/ERS guidelines. Spirometry was repeated after inhalation of $400 \mu \mathrm{g}$ salbutamol administered by puffer and holding chamber, without withholding any long-acting bronchodilators. Reference values used for spirometry and lung volumes 
were those of Quanjer et al. ${ }^{15,16}$ and expressed as z-scores. Airflow obstruction was defined as FEV1/FVC z-score $<$ lower limit of normal, i.e. -1.64 .

\section{Six-Minute Walk Test}

The 6MWT was conducted using a $25 \mathrm{~m}$ straight line walking path, and in accordance with ATS/ERS guidelines, ${ }^{17}$ at enrolment, at the first session of the 16session program of rehabilitation (which was used as the baseline six-minute walk distance, 6MWD), at completion and again at 3 months after completion of rehabilitation.

\section{Forced Oscillation Technique (FOT)}

FOT measurements of respiratory impedance were obtained using an in-house developed device as previously described $^{18,19}$ (see Appendix for further details). Participants were tested sitting upright with their head in a neutral position, wearing a nose clip and supporting their own cheeks and chin to minimise upper airway shunting, breathing through an anti-bacterial/-viral filter (Suregard, Bird Healthcare, Port Melbourne, Australia). After establishing stable tidal breathing; a multiple frequency signal $(5,11,19 \mathrm{~Hz})$ was superimposed onto tidal breathing during a single continuous acquisition of $60 \mathrm{~s}$, which ended with three maximal deep inspirations from which inspiratory capacity (IC) was calculated.

The FOT signals were processed to derive impedance parameters as previously described ${ }^{20}$ for this analysis, we focussed on impedance at $5 \mathrm{~Hz}$, which better reflects small airway function and EFL. Respiratory system resistance and reactance, respectively, were calculated as the mean of all artefact-free tidal breaths $\left(\mathrm{R}_{\text {mean }}, \mathrm{X}_{\text {mean }}\right)$ and also for inspiration only $\left(\mathrm{R}_{\text {insp }}, \mathrm{X}_{\text {insp }}\right)$. DeltaXrs, an index of EFL, was calculated as the mean inspiratory reactances of all breaths, minus the mean expiratory reactances from all breaths. A cutoff value of $\geq 2.8 \mathrm{cmH}_{2}$ O.s. $\mathrm{L}^{-1}$ indicates the presence of EFL. ${ }^{10}$ Reference equations used for FOT parameters were those of Brown et al. ${ }^{21}$ The higher of the two closest IC measurements was used for analysis.

See Appendix for a description of quality control measures. Briefly, the volume-time and resistance-time curves were monitored during acquisition for possible glottic closures, leaks or unusually large or rapid breaths, which, if detected, the acquisition was terminated and repeated. An automated, computerised algorithm was then applied postacquisition to further identify artefacts. ${ }^{22}$ The relevant breath was then removed from the acquisition and a minimum of 5 complete breaths were required for analysis.

\section{Pulmonary Rehabilitation Program}

The pulmonary rehabilitation program comprised 16 sessions of two 1-hr supervised exercise rehabilitation classes per week with a focus on lower limb endurance training, aiming to achieve 30 mins total lower limb endurance training per session either through ground walking, treadmill or exercise bike. Classes also included upper and lower limb functional strength training and upper limb endurance exercises, as well as balance and flexibility training as indicated. There was some program individualisation based on the initial assessment at enrolment.

\section{Statistical Analyses}

Differences in 6MWD, SGRQ and FOT parameters from baseline to completion of rehabilitation and from baseline to the follow-up visit 3 months after rehab completion, were examined by paired t-tests for normally distributed data or Wilcoxon signed-rank tests for non-normally distributed data. The relationships between baseline Rrs and Xrs and; 6MWD and SGRQ were examined by Spearman correlation (because of study cohort size). The relationships between baseline Rrs and Xrs and; changes in 6MWD were similarly examined by linear correlation. A p-value $<0.05$ was considered statistically significant. An expected Spearman correlation coefficient of 0.65 or greater, would be detected in 15 subjects with $80 \%$ power and $5 \%$ type I error rate.

\section{Results}

\section{Subject Characteristics}

The anthropometrics and baseline lung function data of the 15 subjects are provided in Table 1. Participants had moderate airflow obstruction according to spirometry, ${ }^{15} \mathrm{R}_{\text {mean }}$ and $\mathrm{X}_{\text {mean }}{ }^{21}$ (Tables 1 and 2). The cohort had mild hyperinflation and no gas trapping according to the mean FRC/TLC and RV/TLC z-scores ${ }^{16}$ (Table 1), respectively. Eight subjects exhibited EFL according to their DeltaXrs being $\geq 2.8$ $\mathrm{cmH}_{2} \mathrm{O}$.s. $\mathrm{L}^{-1}$. The mean baseline 6MWD was normal but there was a wide range of values (Figure 1A). Quality of life of the cohort as a whole, as measured by mean total SGRQ score, was moderately impaired ${ }^{23}$ (Table 2 ).

Similar to our previous findings, ${ }^{9}$ forced oscillation impedance parameters at baseline related to hyperinflation. $R_{\text {mean }}$, $\mathrm{R}_{\text {insp }}, \mathrm{X}_{\text {mean }}$ and $\mathrm{X}_{\text {insp }}$ correlated with IC and IC/TLC (see Figure 2A and B and Table A1 in the Appendix). Baseline DeltaXrs also correlated with IC/TLC. 
Table I Study Demographics

\begin{tabular}{|l|l|}
\hline N = I5 (8 Males) & Mean(SD) \\
\hline Age (years) & $75.2(6.1)$ \\
BMI (kg/m²) & $24.8(4.1)$ \\
Pack years & $43.5(24.1)$ \\
FEVI (L) & $1.24(0.48)$ \\
FEVI z-score & $-2.61(0.84)$ \\
FVC (L) & $2.99(0.79)$ \\
FVC z-score & $-0.37(1.1 \mathrm{I})$ \\
FEVI/FVC (\%) & $42(0.12)$ \\
FEVI/FVC z-score & $-3.58(0.96)$ \\
IC (L) & $1.72(0.43)$ \\
FRC/TLC z-score & $1.93(1.74)$ \\
RV/TLC z-score & $1.57(1.62)$ \\
\hline
\end{tabular}

Abbreviations: $\mathrm{BMI}$, body mass index; FEVI, forced expiratory volume in I s; FVC, forced vital capacity; IC, inspiratory capacity measured by forced oscillation technique; FRC/TLC, functional residual capacity to total lung capacity ratio; RV/ TLC, residual volume to total lung capacity ratio.

Baseline FOT and spirometry parameters were significantly correlated with SGRQ. $X_{\text {mean }}$ and DeltaXrs correlated with SGRQ (see Figure $3 \mathrm{~A}$ and $\mathrm{B})\left(\mathrm{n}=12, \mathrm{r}_{\mathrm{s}}=-0.75, \mathrm{p}<0.01\right.$; $\mathrm{r}={ }_{\mathrm{s}} 0.80, \mathrm{p}<0.01$, respectively). FVC $\mathrm{z}$-score also correlated with SGRQ (see Figure 3C) $\left(\mathrm{r}_{\mathrm{s}}=-0.73, \mathrm{p}<0.01\right)$. Rrs parameters were not related to SGRQ $\left(\mathrm{R}_{\text {mean }}, \mathrm{r}_{s}=0.43, \mathrm{p}=0.17\right.$, see Figure 3D). At baseline, FOT and spirometry parameters were unrelated to 6MWD.

\section{Findings at Completion of the Pulmonary Rehabilitation Program}

The 6MWD improved at completion of the pulmonary rehabilitation program $(\mathrm{p}=0.005$; see Figure $1 \mathrm{~A}$ and Table 2). $X_{\text {insp }}$ also significantly improved at program completion compared with baseline $(p=0.008$; see Figure $1 \mathrm{~B}$ and Table 2), although there were no changes in any other FOT parameters, IC or SGRQ (see Table 2).

There were no predictors of change in 6MWD at program completion (see Table 3). 6MWD at baseline did not predict its improvement $\left(\mathrm{r}_{\mathrm{s}}=0.18, \mathrm{p}=0.53\right)$; even those subjects whose 6MWD was in the normal range showed increases in exercise capacity. Baseline FOT and spirometry parameters, and their changes at program completion, were unrelated to the changes in 6MWD at program completion (see Figure 4A and C). There was a wide range of time taken to complete the program (range 56-196 days), however, this did not predict changes to 6MWD $(\mathrm{p}=0.39)$.

\section{Findings at 3 Months Post-Completion of Pulmonary Rehabilitation}

At 3 months after program completion, 12 of the 15 subjects returned for follow-up assessment. In those 12 subjects, the FOT parameters, IC and 6MWD at follow up were not different from values at either program completion $(\mathrm{p}=0.11$ for $6 \mathrm{MWD})$ or baseline $(\mathrm{p}=0.27$ for $6 \mathrm{MWD}$; see Table 2 and Figure 4A).

Although there was no change in mean 6MWD between the time of program completion and 3 months later, there was considerable heterogeneity in the changes (see Figure 1A) with continued improvement in some but deterioration in others. This change in 6MWD subsequent to program completion was predicted by the severity of ventilatory impairment at baseline. Greater ventilatory impairment measured by Xrs parameters predicted

Table 2 FOT Impedance Parameters, 6MWD and SGRQ at Baseline, at Completion and 3 Months Post-Completion of Pulmonary Rehabilitation

\begin{tabular}{|c|c|c|c|c|c|}
\hline & Baseline & Rehab Finish & $\mathbf{P}$ & 3 Months Post-Rehab ${ }^{a}$ & $\mathbf{P}^{\mathbf{a}}$ \\
\hline $\mathrm{R}_{\text {mean }}\left(\mathrm{cmH}_{2} \mathrm{O} . \mathrm{s} . \mathrm{L}^{-1}\right)$ & $5.58(1.54)$ & $5.46(1.72)$ & $0.57^{b}$ & $5.57(1.23)$ & 0.70 \\
\hline $\mathrm{R}_{\text {insp }}\left(\mathrm{cmH}_{2}\right.$ O.s. $\left.\mathrm{L}^{-1}\right)$ & $4.40(0.92)$ & $4.30(0.81)$ & 0.49 & $4.38(1.07)$ & 0.81 \\
\hline $\mathrm{X}_{\text {mean }}\left(\mathrm{cmH}_{2}\right.$ O.s. $\left.\mathrm{L}^{-1}\right)$ & $-4.46(2.47)$ & $-4.22(3.03)$ & 0.53 & $-3.91(2.61)$ & 0.52 \\
\hline $\mathrm{X}_{\text {insp }}\left(\mathrm{cmH}_{2}\right.$ O.s. $\left.\mathrm{L}^{-1}\right)$ & $-2.35(1.02)$ & $-2.04(0.85)$ & 0.008 & $-2.38(1.08)$ & 0.84 \\
\hline DeltaXrs $\left(\mathrm{cmH}_{2}\right.$ O.s.L $\left.{ }^{-1}\right)$ & $3.31(2.76)$ & $3.02(3.48)$ & $0.36^{\mathrm{b}}$ & $2.4 I(2.66)$ & $0.18^{b}$ \\
\hline IC (L) & $1.70(0.43)$ & $1.79(0.48)$ & 0.21 & $1.85(0.58)$ & $0.43^{b}$ \\
\hline 6MWD (m) & $398.7(102.8)$ & $450.7(127.8)$ & 0.005 & $406.3(150.9)$ & 0.27 \\
\hline$S_{G R Q}{ }^{c}$ & $41.36(18.05)$ & $34.16(14.24)$ & 0.10 & $38.18(18.99)$ & 0.59 \\
\hline
\end{tabular}

Notes: All values presented as mean(SD) and comparisons are against baseline. ${ }^{a} n=12$ for comparisons between start of pulmonary rehabilitation with follow-up visit at 3 months after completion of pulmonary rehabilitation ( 3 subjects failed to attend follow-up); ${ }^{b}$ Wilcoxon signed-rank test; ${ }^{c} n=12$ due to incomplete SGRQ data; Rehab finish $=$ at completion of pulmonary rehabilitation.

Abbreviations: $P$, p value; 3 months post-rehab, 3 months after completion of rehabilitation program; $R_{\text {mean }}$, mean resistance; $R_{\text {insp }}$, inspiratory resistance; $X_{\text {mean }}$, mean reactance; $\mathrm{X}_{\text {insp }}$, inspiratory reactance; DeltaXrs, expiratory flow limitation measured by forced oscillation technique; IC, inspiratory capacity measured by forced oscillation technique; 6MWD, six-minute walk distance; SGRQ, St. George's Respiratory Questionnaire total score. 

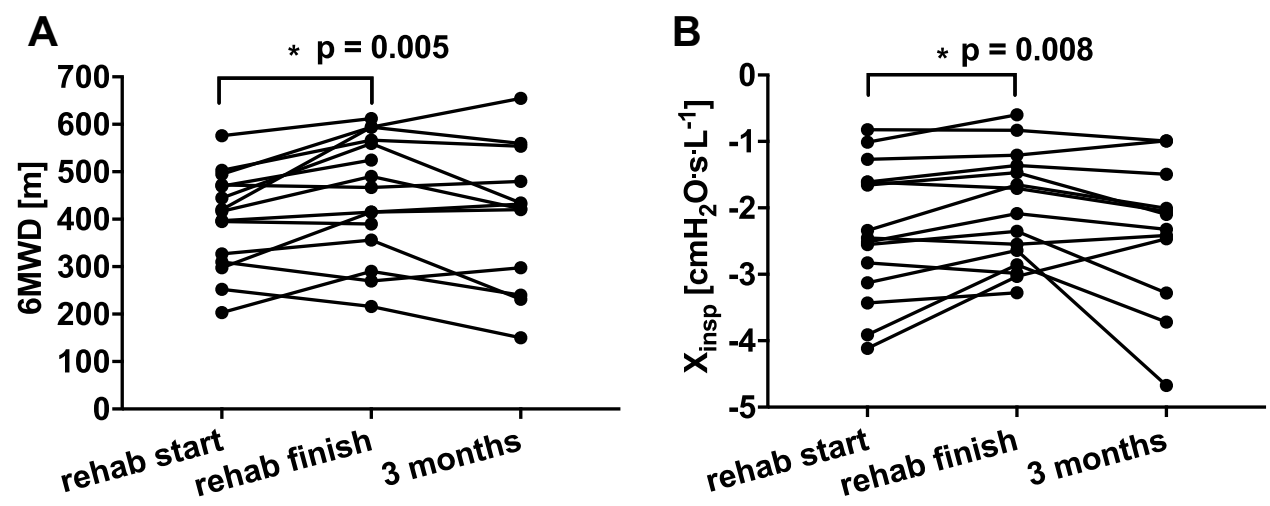

Figure I Changes in (A) six-minute walk distance and (B) inspiratory reactance at 3 time points: baseline, completion of pulmonary rehabilitation and; 3 months after completion of pulmonary rehabilitation. ${ }^{*} \mathrm{p}<0.05$.

Abbreviations: 6MWD, six-minute walk distance; $X_{i n s p}$ inspiratory reactance; Rehab start, at the start of the pulmonary rehabilitation program; Rehab finish, at the completion of the pulmonary rehabilitation program; 3 months, 3 months after completion of the pulmonary rehabilitation program.
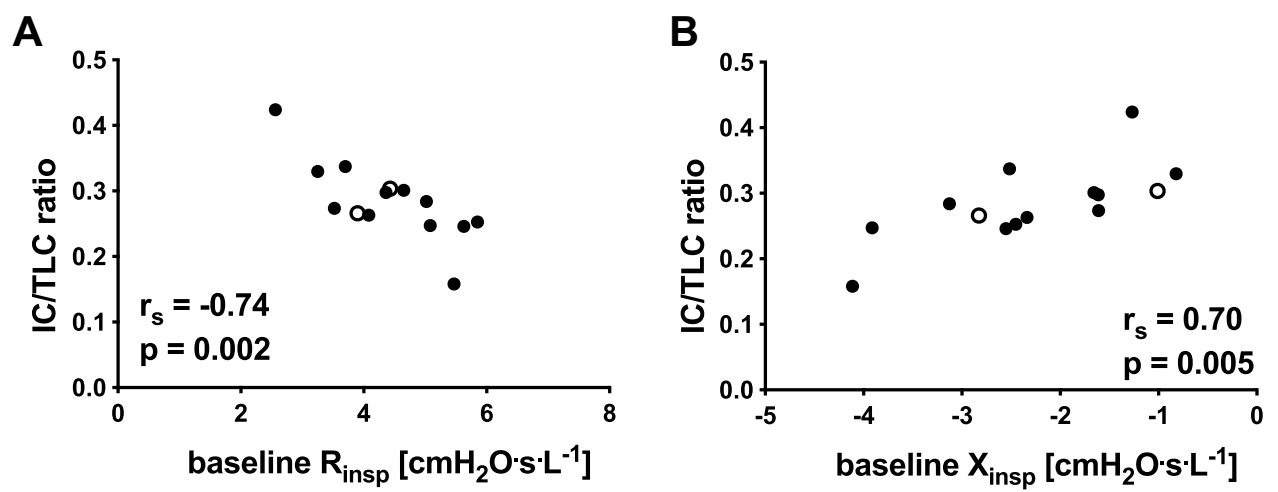

Figure 2 Relationships at baseline between hyperinflation as measured by inspiratory capacity corrected for total lung capacity (IC/TLC) with (A) inspiratory resistance and (B) inspiratory reactance. ${ }^{\circ}$ subject failed to attend follow up at 3 months post-rehabilitation completion ( $\mathrm{n}=14$; I subject unable to perform plethysmographic lung volumes).

Abbreviations: IC/TLC ratio, inspiratory capacity corrected by total lung capacity; $\mathrm{R}_{\text {insp }}$, inspiratory resistance; $\mathrm{X}_{\text {insp }}$, inspiratory reactance; $r_{s}$, Spearman correlation coefficient; $p$, p-value.

a greater decrease in 6MWD over this time (see Table 3). A greater loss in 6MWD at 3 months, was associated with a lower $X_{\text {mean }}\left(r_{s}=0.62, p=0.03\right)$, a higher DeltaXrs (see Figure $4 \mathrm{~B}, \mathrm{r}_{\mathrm{s}}=-0.65, \mathrm{p}=0.02$ ), and a lower FVC z-score (see Figure 4D, $r_{s}=0.62, p=0.03$ ). Baseline Rrs parameters were unrelated to the change in 6MWD.

There was no significant change in $\mathrm{X}_{\text {insp }}$ between completion of pulmonary rehabilitation and 3 months postcompletion $(\mathrm{p}=0.07$, see Figure $1 \mathrm{~B})$.

\section{Discussion}

In this prospective, observational study of 15 COPD subjects attending pulmonary rehabilitation, we found that although baseline Xrs parameters $\left(X_{\text {mean }}\right.$, DeltaXrs $)$ did not correlate with the improvement in 6MWD at the end of the rehabilitation program, they predicted the decrease in 6MWD between program completion and 3 months after completion. This worsening of 6MWD occurred predominantly in those with greater ventilatory impairment as determined by $\mathrm{X}_{\text {mean }}$, DeltaXrs and FVC. Hence, those who had less ventilatory impairment were more likely to maintain their 6MWD. Measures of Xrs ( $\mathrm{X}_{\text {mean }}$, DeltaXrs) but not Rrs correlated with quality of life. Therefore, greater ventilatory impairment in COPD patients as measured by forced oscillatory Xrs parameters, may affect their ability to maintain the improvements in exercise capacity afforded by pulmonary rehabilitation.

It is difficult to predict the improvement in exercise capacity in COPD patients undergoing pulmonary rehabilitation. Several studies have examined potential baseline predictors of response to pulmonary rehabilitation, with variable findings. ${ }^{3-5,24-29}$ Lower FEV1/FVC predicted a greater improvement in $6 \mathrm{MWD}$ in one study, ${ }^{3}$ while a higher FEV1/FVC ratio predicted a better response in another study. ${ }^{5}$ In both of those 
A

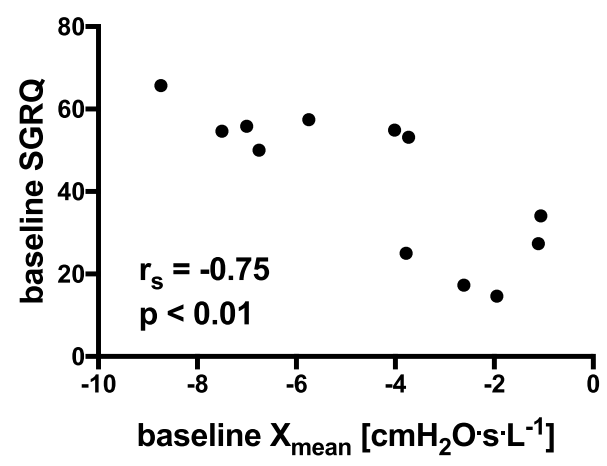

C

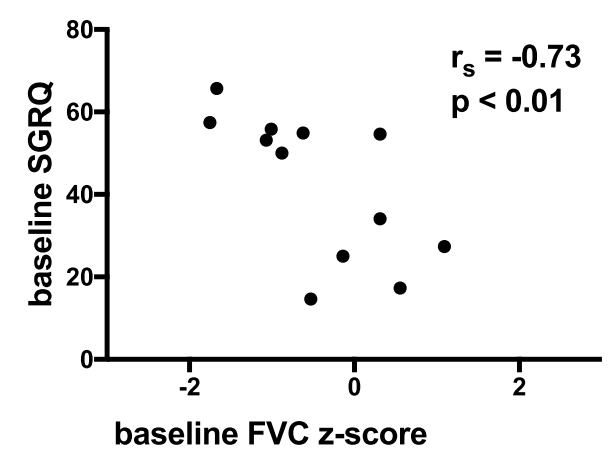

B

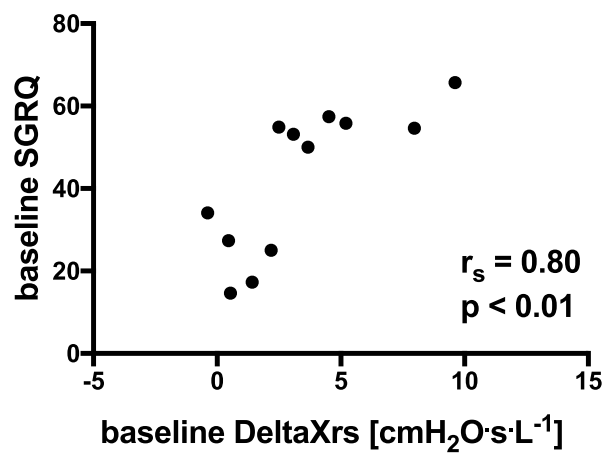

D

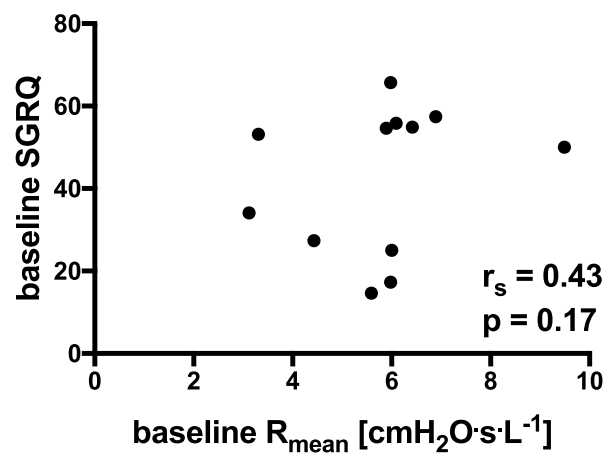

Figure 3 Relationships at baseline between FOT parameters with SGRQ for (A) mean reactance (expressed as absolute values), (B) index of expiratory flow limitation (expressed as absolute values), (C) forced vital capacity (expressed as z-scores) and (D) mean resistance (expressed as absolute values). ( $\mathrm{n}=$ I2; missing SGRQ data in 3 subjects).

Abbreviations: $\mathrm{X}_{\text {mean }}$, mean reactance; FVC, forced vital capacity; DeltaXrs, index of expiratory flow limitation as measured by FOT; $\mathrm{R}_{\text {mean }}$ mean resistance; SGRQ, St. George's Respiratory Questionnaire total score.

studies, a lower baseline 6MWD predicted its greater improvement with rehabilitation. ${ }^{3,5}$ Less breathlessness predicted a better response in one study, ${ }^{4}$ but more breathlessness was predictive in another. ${ }^{28}$ Higher BMI and worse hypoxaemia ${ }^{29}$ have also predicted better response. In the present study, we did not find FOT parameters or spirometry to be predictors of response at program completion, likely due to the small subject numbers. Therefore, at present, it is not possible to predict which COPD patients will show benefit at the end of the pulmonary rehabilitation program.

This is the first study to examine predictors of the changes that occur after the completion of pulmonary rehabilitation. The loss of benefit after program completion is a wellrecognised problem in COPD management and this was observed in some subjects of the present study. There are likely many different factors involved that affect either

Table 3 Correlations Between Baseline FOT Parameters and 6-minute Walk Distance

\begin{tabular}{|l|l|l|l|l|}
\hline & Baseline 6MWD & $\Delta_{\text {comp }}$ 6MWD & $\Delta_{3 \mathbf{M}}$ 6MWD & $\Delta_{\text {comp }}-\Delta_{\mathbf{3}}$ 6MWD \\
\hline $\mathrm{R}_{\text {mean }}$ & $-0.05(0.86)$ & $-0.30(0.27)$ & $-0.62(0.03)$ & $-0.48(0.1 \mathrm{I})$ \\
$\mathrm{R}_{\text {insp }}$ & $0.21(0.44)$ & $-0.17(0.55)$ & $-0.32(0.32)$ & $-0.41(0.18)$ \\
$\mathrm{X}_{\text {mean }}$ & $0.18(0.52)$ & $0.28(0.31)$ & $0.71(0.009)$ & $0.62(0.03)$ \\
$\mathrm{X}_{\text {insp }}$ & $0.16(0.57)$ & $0.37(0.18)$ & $0.64(0.02)$ & $0.43(0.16)$ \\
DeltaXrs & $-0.21(0.45)$ & $0.30(0.30)$ & $-0.73(0.007)$ & $-0.65(0.02)$ \\
\hline
\end{tabular}

Notes: All values presented as Spearman correlation coefficient $r_{s}$ ( $p$-value).

Abbreviations: 6MWD, 6-minute walk distance; $\Delta_{\text {comp }}$, change between baseline and completion of pulmonary rehabilitation; $\Delta_{3 M}$, change between baseline and 3 months post-program completion; $\Delta_{\text {comp }}-\Delta_{3 M}$, change between program completion and 3 months post-completion; $R_{\text {mean }}$, mean resistance; $R_{\text {insp }}$, inspiratory resistance; $X_{\text {mean }}$, mean reactance; $X_{\text {insp }}$, inspiratory reactance; DeltaXrs = expiratory flow limitation index. 
A
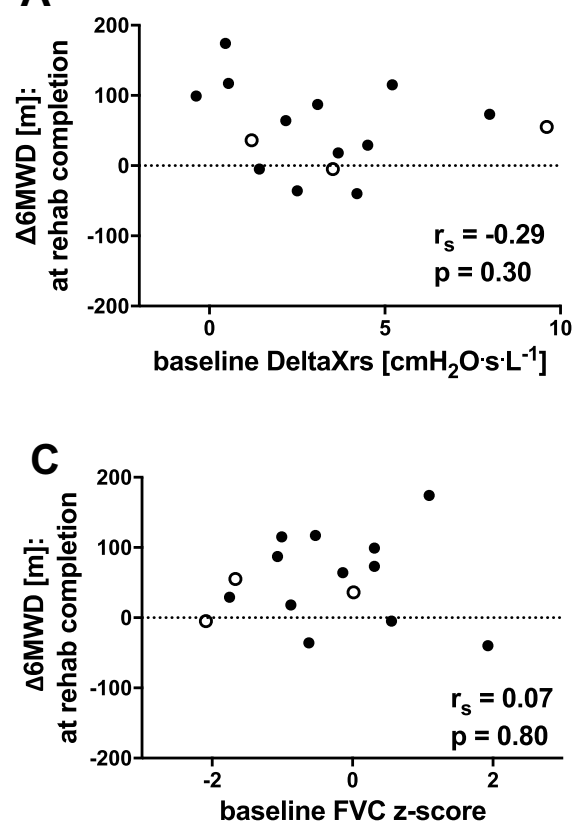

B
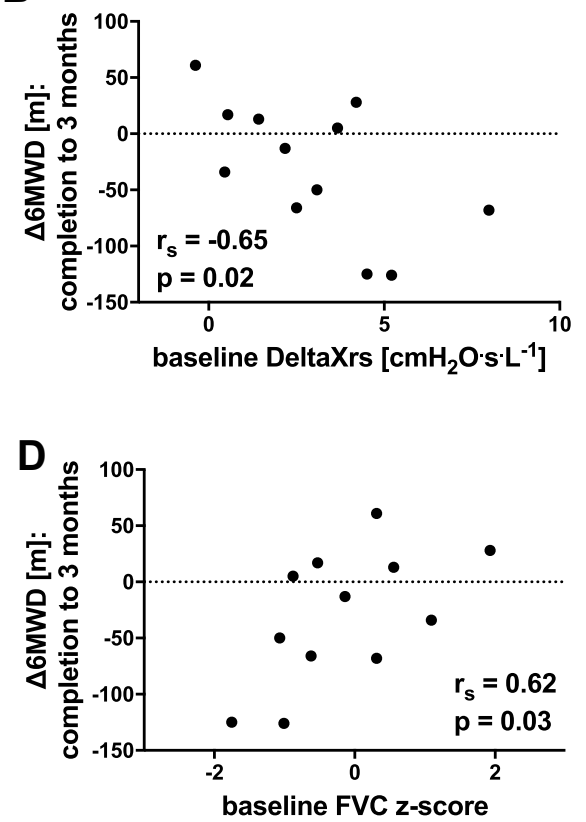

Figure 4 Relationships between changes in exercise capacity ( 6 6MWD) (A) at completion of rehabilitation and baseline DeltaXrs (expressed as absolute values), (B) between completion and 3 months after completion and baseline DeltaXrs (expressed as absolute values); (C) at completion of rehabilitation and baseline FVC z-score, and (D) between completion and 3 months after completion and baseline FVC z-score. 'subject failed to attend follow-up at 3 months post-rehabilitation completion.

Abbreviations: $\triangle 6 \mathrm{MWD}$, change in six-minute walk distance; DeltaXrs, index of expiratory flow limitation as measured by FOT; FVC, forced vital capacity; $r_{s}$, Spearman correlation coefficient; $\mathrm{p}$, $\mathrm{p}$-value.

ongoing improvement or deterioration after leaving supervised exercise. The improvements in 6MWD achieved by the end of the program may be attributed to the motivation and regular sessions provided by the program, hence the severity of ventilatory impairment (measured by Xrs and FVC) may have little influence on those benefits. Indeed, baseline lung function was unrelated to the change in 6MWD at program completion (see Table 3). In contrast, after leaving the supervised environment of the rehabilitation program, the underlying ventilatory impairment may then affect the ability to continue the improvement, or indeed to maintain the benefits gained during training. This may explain the relationships between baseline ventilatory impairment $\left(X_{\text {mean }}\right.$ and DeltaXrs) and change in 6MWD after leaving the program (see Table 3). As well as having a direct effect on an individual's ability to maintain fitness, ventilatory impairment as measured by FOT could also be a marker of morphological changes and extra-pulmonary manifestations in COPD that could affect exercise capacity, e.g. emphysema severity ${ }^{30}$ or reduced muscle function. ${ }^{31}$

Baseline $\mathrm{X}_{\text {mean }}$, DeltaXrs and FVC, which correlated with the change in $6 \mathrm{MWD}$ that occurred between finishing rehabilitation and 3 months later, are lung function measures of ventilatory impairment in COPD. Xrs (and Rrs) correlates strongly with percentage of emphysematous lung, ${ }^{30}$ which is known to be poorly ventilated. Furthermore, Xrs correlates strongly with airway closure (as measured by FVC) in asthmatics during induced bronchoconstriction, ${ }^{32}$ with closing volume in healthy subjects ${ }^{33,34}$ and with the difference between plethysmographic TLC and single breath alveolar volume difference in COPD subjects. ${ }^{35}$ Thus, Xrs is indicative of severe airway narrowing and closure, which increases ventilation heterogeneity. These experimental findings are also consistent with computational modelling studies of FOT $^{36,37}$ examining the effects of airway narrowing and closure on lung impedance. These models predicted that widespread and heterogeneous small airway narrowing and closure would have a large effect on reactance. This is further supported by murine $^{38}$ and porcine ${ }^{39}$ models of lung de-recruitment (closure). It is mechanistically plausible that airway closure during tidal breathing in COPD, that is measured by Xrs, results in sufficient ventilatory impairment that could make it harder to exercise, perhaps by increasing breathlessness. Impairment of Xrs has been correlated with breathlessness in COPD; ${ }^{11,40}$ this then might explain the loss of 6MWD after leaving the pulmonary rehabilitation program, that we observed in the present 
study. This mechanism, however, requires further investigation in future studies.

In the present study, we found that $\mathrm{X}_{\mathrm{insp}}$ significantly improved following pulmonary rehabilitation, whereas $\mathrm{X}_{\text {mean, }}$ DeltaXrs and Rrs measures did not. This change needs to be interpreted cautiously in the absence of a control group. Dynamic expiratory airway collapse may occur in some COPD subjects, which causes severe airway narrowing and formation of choke points, which decreases $X_{\text {exp }}$. The difference between $X_{\text {insp }}$ and $X_{\exp }$ has been shown to be an index of this phenomenon (EFL), ${ }^{10}$ and by inference, dynamic collapse does not

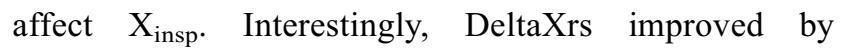
a similar amount (0.31 $\mathrm{cmH}_{2}$ O.L.s ${ }^{-1}$, Table 2) but was not statistically significant, presumably due to greater variation than $X_{\text {insp }}$. The less negative $X_{\text {insp }}$ may indicate that heterogeneous airway narrowing and closure were reduced with pulmonary rehabilitation, i.e. representing improvements in communicating lung volume, ${ }^{41}$ rather than changes to lung elastance. There are no published reports on changes in Rrs or Xrs with pulmonary rehabilitation. In the present study, the changes in $\mathrm{X}_{\text {insp }}$ were not associated with any changes in SGRQ or in 6MWD, and were not explained by changes in tidal volume or breathing frequency. Speculatively, the improvement in $\mathrm{X}_{\text {insp }}$ might be due to increased medication adherence or better mucus clearance with exercise during the rehabilitation program.

Although we found no changes in DeltaXrs with pulmonary rehabilitation, EFL measured by the negative expiratory pressure method improved with rehabilitation in two COPD studies ${ }^{12,42}$ but not in a third. ${ }^{43}$ The negative expiratory pressure technique involves the application of negative pressure during the expiratory phase of tidal breathing, where a lack of increase in flow indicates flow limitation. There was good agreement in detecting expiratory flow limitation between the FOT and negative expiratory pressure methods, ${ }^{44}$ but a suggestion that DeltaXrs was more sensitive in detecting bronchodilator-induced change. Both measurements of EFL are clinically important, however; EFL measured by the negative pressure technique predicts a greater reduction in hyperinflation following bronchodilator. ${ }^{45}$ EFL measured by FOT is associated with worse gas trapping, ${ }^{11}$ worse breathlessness and SGRQ, ${ }^{11,46}$ and recovers in COPD exacerbations with treatment. $^{47,48}$ A relationship between SGRQ and EFL (DeltaXrs) was also found in the present study (see Figure 3B). This relationship is likely due to exercise limitation and worse breathlessness induced by expiratory flow limitation, since exercise leads to increased flow and breathing rate, which in the presence of EFL leads to greater hyperinflation. ${ }^{49}$

Being a small pilot study, the limitations of size mean the results must be interpreted with some caution and need to be replicated. Since we were primarily interested in the potential relationships and predictive value of baseline FOT parameters with changes in 6MWD, rather than in changes with rehabilitation per se, we did not enrol a control group. Any improvements in FOT parameters with pulmonary rehabilitation, i.e. $\mathrm{X}_{\mathrm{insp}}$, should therefore be viewed cautiously. Participants were highly selected by the exclusion of subjects with comorbidities that could have affected 6MWD, e.g. orthopaedic and cardiovascular disease. The loss of 3 patients to follow-up at 3 months post-completion could potentially affect our results, although the Xrs of those 3 subjects were spread over the range of Xrs values of the cohort (see Figures $1 \mathrm{~A}$ and $4 \mathrm{~A}$ ). Inhaled bronchodilators were not withheld prior to lung function testing, and are known to affect inspiratory capacity and exercise tolerance in COPD. ${ }^{50,51}$ Although all 15 participants were taking a long-acting betaagonist and 12/15 subjects were also taking a long-acting anti-muscarinic, it is possible that long-acting bronchodilators could affect the relationship between Xrs parameters with changes in 6MWD and SGRQ.

In conclusion, we found that in COPD patients without significant cardiovascular or orthopaedic comorbidities, Xrs parameters related to respiratory quality of life and to hyperinflation at the start of a pulmonary rehabilitation program. Greater ventilatory impairment indicated by $\mathrm{X}_{\text {mean }}$ and DeltaXrs, related to the change in 6MWD between program completion and 3 months after completion. Therefore, Xrs indices may be clinically useful as a measure of ventilatory impairment in COPD patients attending pulmonary rehabilitation and our results provide a basis for further studies of FOT in the setting of pulmonary rehabilitation for COPD.

\section{Summary at a Glance}

Baseline reactance measures may predict the loss of exercise capacity after completion of pulmonary rehabilitation, in COPD.

\section{Acknowledgements}

We would like to acknowledge the support of the study by Meredith King, Luke Scott and Aimee Fraser as physiotherapists of the pulmonary rehabilitation program and all the patients participating in the study. 


\section{Author Contributions}

All authors contributed to data analysis, drafting or revising the article, gave final approval of the version to be published, and agree to be accountable for all aspects of the work.

\section{Funding}

SCZ was supported by a NHMRC Postgraduate Scholarship. This research did otherwise not receive any specific grant from funding agencies in the public, commercial, or not-forprofit sectors.

\section{Disclosure}

Dr Sabine C Zimmermann was supported by the National Health and Medical Research (NHMRC) Postgraduate Scholarship, during the conduct of the study; non-financial support from Boehringer Ingelheim, Glaxo Smith Kline, Astra Zeneca, Novartis, and Menarini, outside the submitted work. Dr Cindy Thamrin reports a patent WO 2006130922 A1 issued; and has intellectual property arrangements with THORASYS Thoracic Medical Systems Inc and Restech srl relating to scientific collaborations, but does not have any financial relationships with either company. She is also a member of a current international committee on FOT technical standards. Prof. Dr. Gregory King reports grants from NHMRC, non-financial support from Restech, during the conduct of the study; personal fees from Pharmaceutical Industry, GSK, and grants from CycloPharma, outside the submitted work. The authors report no other conflicts of interest in this work.

\section{References}

1. McCarthy B, Casey D, Devane D, Murphy K, Murphy E, Lacasse Y. Pulmonary rehabilitation for chronic obstructive pulmonary disease. Cochrane Database Syst Rev. 2015;2.

2. Schroff P, Hitchcock J, Schumann C, Wells JM, Dransfield MT, Bhatt SP. Pulmonary rehabilitation improves outcomes in chronic obstructive pulmonary disease independent of disease burden. Ann Am Thorac Soc. 2016;14:26-32.

3. Di Meo F, Pedone C, Lubich S, Pizzoli C, Traballesi M, Incalzi RA. Age does not hamper the response to pulmonary rehabilitation of COPD patients. Age Ageing. 2008;37(5):530-535. doi:10.1093/ageing/afn 126

4. Garrod R, Marshall J, Barley E, Jones PW. Predictors of success and failure in pulmonary rehabilitation. Eur Respir J. 2006;27(4):788-794. doi:10.1183/09031936.06.00130605

5. van Ranst D, Otten H, Meijer JW, van 't Hul AJ. Outcome of pulmonary rehabilitation in COPD patients with severely impaired health status. Int J Chron Obstruct Pulmon Dis. 2011;6:647-657. doi:10.2147/COPD.S24579

6. Garcia-Aymerich J, Farrero E, Felez MA, et al. Risk factors of readmission to hospital for a COPD exacerbation: a prospective study. Thorax. 2003;58(2):100-105. doi:10.1136/thorax.58.2.100
7. Garcia-Aymerich J, Lange P, Benet M, Schnohr P, Antó JM. Regular physical activity reduces hospital admission and mortality in chronic obstructive pulmonary disease: a population based cohort study. Thorax. 2006;61(9):772-778. doi:10.1136/thx.2006.060145

8. Garcia-Rio F, Rojo B, Casitas R, et al. Prognostic value of the objective measurement of daily physical activity in patients with COPD. Chest. 2012;142(2):338-346. doi:10.1378/chest.11-2014

9. Milne S, Hammans C, Watson S, Farah CS, Thamrin C, King GG. Bronchodilator responses in respiratory impedance, hyperinflation and gas trapping in COPD. COPD. 2018;15:1-9.

10. Dellacà RL, Santus $P$, Aliverti A, et al. Detection of expiratory flow limitation in COPD using the forced oscillation technique. Eur Respir J. 2004;23(2):232-240. doi:10.1183/09031936.04.00046804

11. Dean J, Kolsum U, Hitchen P, Gupta V, Singh D. Clinical characteristics of COPD patients with tidal expiratory flow limitation. Int $J$ Chron Obstruct Pulmon Dis. 2017;12:1503-1506. doi:10.2147/COPD.S137865

12. Yoshimi K, Ueki J, Seyama K, et al. Pulmonary rehabilitation program including respiratory conditioning for chronic obstructive pulmonary disease (COPD): improved hyperinflation and expiratory flow during tidal breathing. $J$ Thorac Dis. 2012;4(3):259-264. doi:10.3978/j.issn.2072-1439.2012.03.17

13. Casaburi R, Porszasz J, Burns MR, Carithers ER, Chang RS, Cooper CB. Physiologic benefits of exercise training in rehabilitation of patients with severe chronic obstructive pulmonary disease. Am J Respir Crit Care Med. 1997;155(5):1541-1551. doi:10.1164/ajrccm.155.5.9154855

14. Yang IA, Brown JL, George J, et al. COPD-X Australian and New Zealand guidelines for the diagnosis and management of chronic obstructive pulmonary disease: 2017 update. Med J Aust. 2017;207 (10):436-442. doi:10.5694/mja2.2017.207.issue-10

15. Quanjer PH, Stanojevic S, Cole TJ, et al. Multi-ethnic reference values for spirometry for the 3-95 year age range: the global lung function 2012 equations. Eur Respir J. 2012;40:1324-1343. doi:10.1183/09031936.00080312

16. Quanjer PH, Tammeling GJ, Cotes JE, Pedersen OF, Peslin R, Yernault J-C. Lung volumes and forced ventilatory flows. Eur Respir J. 1993;6(Suppl 16):5-40. doi:10.1183/09041950.005s1693

17. Holland AE, Spruit MA, Troosters T, et al. An official European Respiratory Society/American Thoracic Society technical standard: field walking tests in chronic respiratory disease. Eur Respir J. 2014;44(6):1428-1446. doi:10.1183/09031936.00150314

18. Thorpe CW, Salome CM, Berend N, King GG. Modeling airway resistance dynamics after tidal and deep inspirations. J Appl Physiol. 2004;97(5):1643-1653. doi:10.1152/japplphysiol.01300.2003

19. Timmins SC, Coatsworth N, Palnitkar G, et al. Day-to-day variability of oscillatory impedance and spirometry in asthma and COPD. Respir Physiol Neurobiol. 2013;185(2):416-424. doi:10.1016/j.resp.2012.08.017

20. Diba C, GG K, Berend N, Salome CM. Improved respiratory system conductance following bronchodilator predicts reduced exertional dyspnoea. Respir Med. 2011;105(9):1345-1351. doi:10.1016/j. rmed.2011.03.013

21. Brown NJ, Xuan W, Salome CM, et al. Reference equations for respiratory system resistance and reactance in adults. Respir Physiol Neurobiol. 2010;172:162-168. doi:10.1016/j.resp.2010.05.013

22. Robinson PD, Turner M, Brown NJ, et al. Procedures to improve the repeatability of forced oscillation measurements in school-aged children. Respir Physiol Neurobiol. 2011;177(2):199-206. doi:10.1016/j.resp.2011.02.004

23. Müllerova H, Gelhorn H, Wilson H, et al. St George's respiratory questionnaire score predicts outcomes in patients with COPD: analysis of individual patient data in the COPD biomarkers qualification consortium database. Chronic Obstructive Pulm Dis. 2017;4 (2):141-149. doi:10.15326/jcopdf.4.2.2017.0131

24. Altenburg WA, de Greef MHG, Ten Hacken NHT, Wempe JB. A better response in exercise capacity after pulmonary rehabilitation in more severe COPD patients. Respir Med. 2012;106(5):694-700. doi:10.1016/j.rmed.2011.11.008 
25. Crisafulli E, Costi S, Luppi F, et al. Role of comorbidities in a cohort of patients with COPD undergoing pulmonary rehabilitation. Thorax. 2008;63(6):487-492. doi:10.1136/thx.2007.086371

26. Plankeel JF, McMullen B, MacIntyre NR. Exercise outcomes after pulmonary rehabilitation depend on the initial mechanism of exercise limitation among non-oxygen-dependent COPD patients. Chest. 2005;127(1):110-116. doi:10.1378/chest.127.1.110

27. Selzler A-M, Simmonds L, Rodgers WM, Wong EYL, Stickland MK. Pulmonary rehabilitation in chronic obstructive pulmonary disease: predictors of program completion and success. COPD. 2012;9 (5):538-545. doi:10.3109/15412555.2012.705365

28. Spruit MA, Augustin IML, Vanfleteren LE, et al. Differential response to pulmonary rehabilitation in COPD: multidimensional profiling. Eur Respir J. 2015;46(6):1625-1635. doi:10.1183/13993003.00350-2015

29. Vagaggini B, Costa F, Antonelli S, et al. Clinical predictors of the efficacy of a pulmonary rehabilitation programme in patients with COPD. Respir Med. 2009;103(8):1224-1230. doi:10.1016/j.rmed.2009.01.023

30. Karayama M, Inui N, Mori K, et al. Respiratory impedance is correlated with morphological changes in the lungs on three-dimensional CT in patients with COPD. Sci Rep. 2017;7:41709. doi:10.1038/srep41709

31. Donaldson AV, Maddocks M, Martolini D, Polkey MI, Man WDC. Muscle function in COPD: a complex interplay. Int J Chron Obstruct Pulmon Dis. 2012;7:523-535. doi:10.2147/COPD.S28247

32. Downie SR, Salome CM, Verbanck S, Thompson BR, Berend N, King GG. Effect of methacholine on peripheral lung mechanics and ventilation heterogeneity in asthma. J Appl Physiol. 2013;114 (6):770-777. doi:10.1152/japplphysiol.01198.2012

33. Mahadev S, Salome C, Berend N, King G. The effects of low lung volume on airway function in obesity. Respir Physiol Neurobiol. 2013;188(2):192-199. doi:10.1016/j.resp.2013.05.021

34. Nilsen K, Thien F, Thamrin C, et al. Early onset of airway derecruitment assessed using the forced oscillation technique in subjects with asthma. J Appl Physiol. 2019;126(5):1399-1408. doi:10.1152/ japplphysiol.00534.2018

35. Milne S, Jetmalani K, Chapman DG, et al. Respiratory system reactance reflects communicating lung volume in chronic obstructive pulmonary disease. J Appl Physiol. 2019;126(5):1223-1231. doi:10.1152/japplphysiol.00503.2018

36. Gillis HL, Lutchen KR. Airway remodeling in asthma amplifies heterogeneities in smooth muscle shortening causing hyperresponsiveness. J Appl Physiol. 1999;86(6):2001-2012. doi:10.1152/jappl.1999.86.6.2001

37. Thorpe CW, Bates JH. Effect of stochastic heterogeneity on lung impedance during acute bronchoconstriction: a model analysis. J Appl Physiol. 1997;82(5):1616-1625. doi:10.1152/jappl.1997.82.5.1616

38. Lundblad LKA, Thompson-Figueroa J, Allen GB, et al. Airway hyperresponsiveness in allergically inflamed mice: the role of airway closure. Am J Respir Crit Care Med. 2007;175(8):768-774. doi:10.1164/rccm.200610-1410OC
39. Dellaca RL, Andersson Olerud M, Zannin E, et al. Lung recruitment assessed by total respiratory system input reactance. Intensive Care Med. 2009;35(12):2164-2172. doi:10.1007/s00134-009-1673-3

40. Aarli BB, Calverley PMA, Jensen RL, Eagan TML, Bakke PS, Hardie JA. Variability of within-breath reactance in COPD patients and its association with dyspnoea. Eur Respir J. 2015;45(3):625-634. doi:10.1183/09031936.00051214

41. Milne S, Jetmalani K, Chapman DG, et al. Respiratory system reactance reflects communicating lung volume in chronic obstructive pulmonary disease. J Appl Physiol. 2019;1985.

42. Theodorakopoulou EP, Gennimata SA, Harikiopoulou M, et al. Effect of pulmonary rehabilitation on tidal expiratory flow limitation at rest and during exercise in COPD patients. Respir Physiol Neurobiol. 2017;238:47-54. doi:10.1016/j.resp.2017.01.008

43. Chen R, Chen R, Chen X, Chen L. Effect of endurance training on expiratory flow limitation and dynamic hyperinflation in patients with stable chronic obstructive pulmonary disease. Intern Med J. 2014;44 (8):791-800. doi:10.1111/imj.2014.44.issue-8

44. Dellaca RL, Duffy N, Pompilio PP, et al. Expiratory flow limitation detected by forced oscillation and negative expiratory pressure. Eur Respir J. 2007;29(2):363-374. doi:10.1183/09031936.00038006

45. Tantucci C, Duguet A, Similowski T, Zelter M, Derenne J, MilicEmili J. Effect of salbutamol on dynamic hyperinflation in chronic obstructive pulmonary disease patients. Eur Respir J. 1998;12 (4):799-804. doi:10.1183/09031936.98.12040799

46. Haruna A, Oga T, Muro S, et al. Relationship between peripheral airway function and patient-reported outcomes in COPD: a cross-sectional study. BMC Pulm Med. 2010;10(1):10. doi:10.1186/ 1471-2466-10-10

47. Jetmalani K, Timmins SC, Brown NJ, et al. Expiratory flow limitation relates to symptoms during COPD exacerbations requiring hospital admission. Int $J$ Chron Obstruct Pulmon Dis. 2015;10 (1):939-945. doi:10.2147/COPD

48. Johnson MK, Birch M, Carter R, Kinsella J, Stevenson RD. Measurement of physiological recovery from exacerbation of chronic obstructive pulmonary disease using within-breath forced oscillometry. Thorax. 2007;62(4):299-306. doi:10.1136/thx.2006.061044

49. O’Donnell DE, Revill SM, Webb KA. Dynamic hyperinflation and exercise intolerance in chronic obstructive pulmonary disease. Am J Respir Crit Care Med. 2001;164(5):770-777. doi:10.1164/ ajrccm.164.5.2012122

50. Casaburi R, Porszasz J. Reduction of hyperinflation by pharmacologic and other interventions. Proc Am Soc. 2006;3(2):185-189.

51. Di Marco F, Sotgiu G, Santus P, et al. Long-acting bronchodilators improve exercise capacity in COPD patients: a systematic review and meta-analysis. Respir Res. 2018;19:18. doi:10.1186/s12931-0180721-3

\section{Publish your work in this journal}

The International Journal of COPD is an international, peer-reviewed journal of therapeutics and pharmacology focusing on concise rapid reporting of clinical studies and reviews in COPD. Special focus is given to the pathophysiological processes underlying the disease, intervention programs, patient focused education, and self management protocols. This journal is indexed on PubMed Central, MedLine and CAS. The manuscript management system is completely online and includes a very quick and fair peer-review system, which is all easy to use. Visit http://www.dovepress.com/testimonials.php to read real quotes from published authors. 